\title{
Estudio Volumétrico, Viscosimétrico y Termodinámico de DL- Alanina en Soluciones Acuosas de Sulfato de sodio a diferentes Temperaturas
}

\author{
Manuel S. Páez ${ }^{1,2}$, Dairo E. Pérez ${ }^{1,2}$ y Jennifer Lafont ${ }^{1}$ \\ (1) Facultad de Ciencias Básicas, Dpto. de Química, Universidad de Córdoba, Montería-Colombia \\ (2) Grupo de Investigación Fisicoquímica de Mezclas Líquidas, Universidad de Córdoba, Montería-Colombia \\ (e-mail: dairops17@gmail.com)
}

Recibido Jun. 1, 2018; Aceptado Ago. 2, 2018; Versión final Sep. 16, 2018, Publicado Abr. 2019

\begin{abstract}
Resumen
Se han determinado los volúmenes molares aparentes y la viscosidad de la DL-alanina en soluciones acuosas de sulfato de sodio, $\mathrm{Na}_{2} \mathrm{SO}_{4}$, a diferentes molalidades y en el intervalo de temperatura (283 a 308) $\mathrm{K}$ a cada 5K a partir de las medidas de densidad y tiempos de flujo, usando un densímetro de tubo vibratorio Anton Paar DMA 5000 y un viscosímetro automático AMVn, respectivamente. A partir de estos datos, se calcularon: volúmenes molares aparentes límites y aparentes límites de transferencia, números de hidratación, coeficientes de viscosidad de la ecuación Jones-Dole, y parámetros de activación termodinámicos del flujo viscoso. Los resultados indican que el aminoácido se comporta como un soluto formador de la estructura del solvente, seguido de un aumento de la estructura global del agua. Se concluye que la formación del estado de transición va acompañado de una formación de enlaces intermoleculares y una estructura más ordenada de las especies en el estado activado.
\end{abstract}

Palabras clave: volumen molar aparente; dl-alanina; viscosidad; soluciones acuosas; parámetros de activación.

\section{Volumetric, Viscosimetric and Thermodynamic study of Alanine in Aqueous solutions of Sodium Sulphate at different Temperatures}

\begin{abstract}
The apparent molar volumes and viscosity of $\mathrm{DL}$-alanine in aqueous solutions of sodium sulfate, $\mathrm{Na}_{2} \mathrm{SO}_{4}$, at different molalities and in the temperature range (283 to 308$) \mathrm{K}$ at each $5 \mathrm{~K}$ were determined from density measurements and flow times, using a vibratory tube densimeter Anton Paar DMA 5000 and an automatic viscometer $A M V n$, respectively. From these data, the: limiting apparent molar volumes and limiting apparent volumes of transfer, hydration numbers, viscosity coefficients of the Jones-Dole equation, and thermodynamic activation parameters of the viscous flow were calculated. The results indicate that the amino acid behaves as a structure-forming solute on the structure of the solvent, followed by an increase in the overall structure of the water. It is concluded that the formation of the transition state is accompanied by the establishment of intermolecular bonds and a more orderly structure of the species in the activated state.
\end{abstract}

Keywords: apparent molar volume; dl-alanine; viscosity; aqueous solutions; activation parameters. 


\section{INTRODUCCIÓN}

La investigación de las propiedades volumétricas, viscosimétricas y termodinámicas de aminoácidos en disolventes mixtos o pseudobinarios resulta un tema de gran interés, debido a que diversos procesos biológicos que implican cambios en las propiedades físicas, bioquímicas, biomédicas e industrialmente útiles de estos compuestos, necesitan para su completa comprensión una idea adecuada del estado y el comportamiento de estas moléculas en el medio (Romero y otros 2017). Además de su importancia en la industria química y farmacéutica, los aminoácidos también se consideran compuestos modelo los cuales en lugar de las proteínas, en presencia de soluciones acuosas salinas permiten obtener información termodinámica relevante, que ayuda a explicar la desnaturalización y la disociación de las proteínas en unidades secundarias y como también su estabilidad en soluciones acuosas (Rajagopal 2009)

Algunos aminoácidos en presencia de soluciones acuosas salinas, $\mathrm{NaBr}$ y $\mathrm{KBr}$ (Partha y otros 2018), $\mathrm{NaCl}$ (Romero 2017) $\mathrm{MgCl}_{2}$ (Karanth 2013), están disponibles en la literatura. Sin embargo, a pesar de que el estudio del aminoácido DL-alanina en presencia de sulfato de sodio acuoso se ha realizado para informar por la solubilidad y propiedades de transferencia derivadas (Ramasami 2002), volumen molar parcial y compresibilidad adiabática (Wadi 1997), entalpía y volumen molar de transferencia (Liu 2009), este estudio sólo se ha hecho para cubrir a lo sumo tres temperaturas. En adición, también se ha informado que el sulfato de sodio tiene un ligero efecto salino sobre el grupo de péptidos y un fuerte efecto salino sobre grupos no polares (Pica y Graziano 2015). Estos resultados conducen al hecho de que el sulfato de sodio estabiliza la estructura nativa de las proteínas y, por lo tanto, se distingue de los desnaturalizantes de proteínas habituales (Ramasami 2002).

En el presente artículo se reportan datos volumétricos, viscosimétricos y termodinámicos de DL-alanina en soluciones acuosas de sulfato de sodio a diferentes temperaturas, los cuales se discuten en términos de las interacciones dominantes soluto-soluto y soluto-solvente que ocurren en el sistema pseudobinario, en adición a la tendencia de formación/disrupción de la estructura del solvente causada por el soluto en el medio dado. Finalmente, los datos resultantes se interpretan en términos de hidratación de las partes hidrofóbicas e hidrofílicas de la DL-alanina.

\section{MATERIALES Y MÉTODOS}

Se empleó DL-alanina grado analítico $\geq 99.0 \%$ y sulfato de sodio anhidro con pureza de $99.0 \%$, adquiridos de la casa comercial Merck. Antes de su uso la DL-alanina fue recristalizada en soluciones acuosas de etanol y secada al vacío sobre $\mathrm{P}_{2} \mathrm{O}_{5}$. El agua usada para preparar las soluciones fue bidestilada y desgasificada y presentó una conductividad menor que $2.0 \mu \mathrm{S} \cdot \mathrm{cm}^{-1}$. Todas las soluciones se prepararon por pesada en la escala de molalidad, usando un balanza Precisa ES 225SM-DR con una sensibilidad de $\pm 1 \times 10^{-5} \mathrm{~g}$, en botellas herméticamente cerradas para evitar pérdida por evaporación de alguno de los componentes.

En este trabajo, se prepararon cinco solventes pseudobinarios (agua $\left.+\mathrm{Na}_{2} \mathrm{SO}_{4}\right)$, con una concentración en la escala molal respecto a la sal dada entre paréntesis: I $(0.2002 \mathrm{~m})$, II $(0.5998 \mathrm{~m})$, III $(0.9005 \mathrm{~m})$, IV $(1.2000 \mathrm{~m})$ y V $(1.6001 \mathrm{~m})$, para ser mezclado con cantidades apropiadas de DL-Alanina. La incertidumbre en la molalidad fue de $0.0002 \mathrm{~mol} \cdot \mathrm{kg}^{-1}$. La densidad y la viscosidad de las soluciones se midieron con un densímetro digital de tubo vibratorio Anton Paar DMA5000 con una incertidumbre de $\pm 1 \times 10^{-6} \mathrm{~g} \cdot \mathrm{cm}^{-3}$ y un Microviscosímetro automático Anton Paar ${ }^{\circledR}(\mathrm{AMVn})$ con una incertidumbre de $\pm 0.001 \mathrm{mPa} \cdot \mathrm{s}$, respectivamente, a presión atmosférica $0.101 \mathrm{MPa}$ y a las temperaturas de $(283.15,298.15,293.15,298.15,303.15$ y 308.15$) \mathrm{K}$. El densímetro y el viscosímetro fueron calibrados con agua bidestilada a las temperaturas seleccionadas. Los valores de densidad y viscosidad del agua utilizada en la calibración a cada temperatura se tomaron de la literatura (Riddick 1971).

\section{RESULTADOS Y DISCUSIÓN}

Los valores de densidad $(\rho)$ y viscosidad relativa $\left(\eta_{r}=\eta / \eta_{0}\right)$ de las mezclas DL-alanina en los cinco solventes pseudobinarios agua $+\mathrm{Na}_{2} \mathrm{SO}_{4}$ en función de la concentración del aminoácido a las temperaturas de (283.15 - 308.15) K, son reportados en la Tabla 1. Los resultados de las mediciones de densidad ( $\rho$ ) de la DL-alanina en solución acuosas de sulfato de sodio a las temperaturas de 283.15 hasta $308.15 \mathrm{~K}$ se utilizaron para determinar los volumenes molares aparentes $\left(\mathrm{V}_{\varphi}\right)$ del aminoácido en las diferentes soluciones acuosas de $\mathrm{Na}_{2} \mathrm{SO}_{4}$ a las temperaturas de estudio, usando la ecuación (1).

$$
\mathrm{V}_{\varphi}=\frac{\mathrm{M}}{\rho}-\frac{1000\left(\rho-\rho_{0}\right)}{\mathrm{m} \rho \rho_{0}}
$$


Tabla 1: Valores experimentales de la densidad y viscosidad relativa para la mezcla pseudobinaria $\mathrm{DL}-\mathrm{Alanina}+\mathrm{Na}_{2} \mathrm{SO}_{4}$ acuoso a diferentes temperaturas.

\begin{tabular}{|c|c|c|c|c|c|c|c|c|c|c|c|c|}
\hline \multicolumn{13}{|c|}{ DL-Alanina + I [ $\left.\mathrm{H}_{2} \mathrm{O}+\mathrm{Na}_{2} \mathrm{SO}_{4}(0.2002 \mathrm{~m})\right]$} \\
\hline $\mathrm{T} / \mathrm{K}$ & 283.15 & 288.15 & 293.15 & 298.15 & 303.15 & 308.15 & 283.15 & 288.15 & 293.15 & 298.15 & 303.15 & 308.15 \\
\hline$m\left(\mathrm{~mol} \mathrm{Kg}^{-1}\right)$ & \multicolumn{6}{|c|}{$\rho\left(\mathrm{g} \cdot \mathrm{cm}^{-3}\right)$} & \multicolumn{6}{|c|}{]$_{T}$} \\
\hline 0.0000 & 1.02598 & 1.02511 & 1.02387 & 1.02246 & 1.02086 & 1.01907 & 1.000 & 1.000 & 1.000 & 1.000 & 1.000 & 1.000 \\
\hline 0.0300 & 1.02666 & 1.02573 & 1.02448 & 1.02306 & 1.02146 & 1.01967 & 1.010 & 1.009 & 1.008 & 1.008 & 1.007 & 1.008 \\
\hline 0.0504 & 1.02715 & 1.02619 & 1.02494 & 1.02353 & 1.02192 & 1.02012 & 1.016 & 1.015 & 1.014 & 1.014 & 1.012 & 1.013 \\
\hline 0.0763 & .02781 & 1.02686 & 1.02558 & 1.02416 & 1.02255 & 1.02075 & 1.024 & 1.023 & 1.022 & 1.021 & 1.018 & 1.020 \\
\hline 0.1002 & 1.02844 & 1.02747 & 1.02621 & 1.02479 & 1.02317 & 1.02137 & 1.032 & 1.030 & 1.029 & 1.028 & 1.024 & 1.026 \\
\hline 0.2000 & 1.03117 & 1.03014 & 1.02884 & 1.02740 & 1.02577 & 1.02394 & 1.064 & 1.060 & 1.058 & 1.055 & 1.049 & 1.051 \\
\hline 0.4005 & .03658 & 1.03561 & 1.03432 & 1.03284 & 1.03119 & 1.02935 & 1.127 & 1.120 & 1.116 & 1.111 & 1.097 & 1.103 \\
\hline 0.6014 & 1.04163 & 1.04060 & 1.03929 & 1.03777 & 1.03610 & 1.03421 & 1.191 & 1.180 & 1.174 & 1.166 & 1.149 & 1.152 \\
\hline 0.8001 & 1.04646 & 1.04535 & 1.04401 & 1.04246 & 1.04075 & 1.03884 & 1.254 & 1.239 & 1.232 & 1.222 & 1.200 & 1.201 \\
\hline 0000 & & 1.04999 & 1.04859 & 1.04701 & 1.04527 & 1.04333 & 1.317 & 1.299 & 1.290 & 1.276 & 1.254 & 1.248 \\
\hline \multicolumn{13}{|c|}{ DL-Alanina $+\mathrm{II}\left[\mathrm{H}_{2} \mathrm{O}+\mathrm{Na}_{2} \mathrm{SO}_{4}(0.5998 \mathrm{~m})\right]$} \\
\hline $\mathrm{T} / \mathrm{K}$ & 283.15 & 288.15 & 293.15 & 298.15 & 303.15 & 308.15 & 283.15 & 288.15 & 293.15 & 298.15 & 303.15 & 308.15 \\
\hline$m\left(\mathrm{~mol} \mathrm{Kg}^{-1}\right)$ & \multicolumn{6}{|c|}{$\rho\left(\mathrm{g} \cdot \mathrm{cm}^{-3}\right)$} & \multicolumn{6}{|c|}{$\eta_{\mathrm{r}}$} \\
\hline 0.0000 & 07430 & 1.07287 & 1.07126 & 1.06949 & 1.06757 & 1.06550 & 1.000 & 1.000 & 1.000 & 1.000 & 1.000 & 1.000 \\
\hline 0.0318 & 07498 & 1.07355 & 1.07193 & 1.07016 & 1.06823 & & 1.011 & 1.011 & 1.010 & 1.010 & 1.010 & 1.010 \\
\hline 0.0507 & 07540 & 1.07397 & 1.07234 & 1.07057 & 1.06864 & 1.06656 & 1.018 & 1.017 & 1.017 & 1.016 & 1.016 & 1.015 \\
\hline 791 & 07604 & 1.07460 & 1.07297 & & 926 & & 1.028 & 1.027 & 1.026 & 1.025 & 1.025 & 1.023 \\
\hline 997 & 07651 & 1.07507 & 1.07343 & & 1.06971 & & 1.036 & 1.034 & 1.033 & 1.031 & 1.031 & 1.029 \\
\hline 0.2005 & 07882 & 1.07736 & 1.07571 & 393 & 1.07198 & & 1.072 & 1.069 & 1.066 & 1.063 & 1.062 & 1.060 \\
\hline 000 & & 1.08194 & 1.08026 & & & & 1.143 & 138 & 1.133 & 27 & 1.124 & 1.119 \\
\hline & & 1.08622 & 1.08450 & & & & 1.214 & 1.206 & 1.199 & 1.1 & & 1.180 \\
\hline 999 & 09181 & 1.09026 & 1.08853 & & 1.08468 & & 1.285 & 1.274 & 1.265 & 1.253 & 1.247 & 1.239 \\
\hline 0.9985 & .09567 & 1.09410 & 1.09233 & 1.09046 & 1.08851 & 1.08642 & 1.356 & 1.343 & 1.331 & 1.315 & 1.309 & 1.298 \\
\hline \multicolumn{13}{|c|}{ DL-Alanina + III $\left[\mathrm{H}_{2} \mathrm{O}+\mathrm{Na}_{2} \mathrm{SO}_{4}(0.9005 \mathrm{~m})\right]$} \\
\hline $\mathrm{T} / \mathrm{K}$ & 83.15 & 288.15 & 293.15 & 298.15 & 303.15 & 308.15 & 283.15 & 288.15 & 293.15 & 298.15 & 303.15 & 308.15 \\
\hline$m\left(\mathrm{~mol} \mathrm{Kg}^{-1}\right.$ & \multicolumn{6}{|c|}{$\rho\left(\mathrm{g} \cdot \mathrm{cm}^{-3}\right)$} & \multicolumn{6}{|c|}{$\eta_{r}$} \\
\hline 0.0000 & & & 1.10490 & 1.10292 & & & 1.000 & 1.000 & 1.000 & 1.000 & 1.000 & 1.000 \\
\hline & & & 10566 & & & & 1.012 & 111 & 1.011 & & & 1.010 \\
\hline 0.0506 & & 1.10793 & 1.10607 & & 1.1 & & 1.019 & 1.018 & 1.017 & 17 & 1.017 & 1.016 \\
\hline 808 & & 1.10855 & 1.10669 & & & & 1.030 & 30 & 1.028 & & 26 & 1.026 \\
\hline & & & 710 & & & & & 36 & 1.035 & & & 1.031 \\
\hline 005 & & 096 & 10905 & & & & 1.0 & 1.073 & 1.070 & 1.067 & & 1.064 \\
\hline 4001 & 11640 & 1.11464 & 1.11272 & 1.1 & 850 & & 1.151 & 1.145 & 1.140 & 1.134 & 1.130 & 1.127 \\
\hline & & & & & & & & & & & & 1.189 \\
\hline & & & 1.11966 & & & & 1.302 & 1.290 & 1.279 & 68 & & 1.252 \\
\hline & & & 1.12285 & 1.12081 & 1.11855 & 1.11639 & 1.377 & 1.362 & 1.349 & 1.335 & 1.324 & 1.316 \\
\hline \multicolumn{13}{|c|}{ DL-Alanina + IV $\left[\mathrm{H}_{2} \mathrm{O}+\mathrm{Na}_{2} \mathrm{SO}_{4}(1.2000 \mathrm{~m})\right]$} \\
\hline $\mathrm{T} / \mathrm{K}$ & 33.15 & 288.15 & 293.15 & 298.15 & 303.15 & 308.15 & 283.15 & 288.15 & 293.15 & 298.15 & 303.15 & 308.15 \\
\hline$m\left(\mathrm{~mol} \mathrm{Kg}^{-1}\right)$ & & & $\rho(g$. & $\left.m^{-3}\right)$ & & & & & & & & \\
\hline 0.0000 & & & 1.13637 & 1.13420 & & & 1.000 & 1.000 & 1.000 & 1.000 & 1.000 & 1.000 \\
\hline & & & & & & & & & & & & 1.010 \\
\hline & & & 750 & & & & 1.019 & 18 & .017 & & 1.016 & 1.016 \\
\hline 816 & & 1.14025 & 13820 & & & & 1.030 & 1.028 & 1.028 & & 1.026 & 1.025 \\
\hline & & & 1.13855 & & & & & & & & & 1.031 \\
\hline & & & 022 & & & & 1.075 & 69 & 1.069 & 66 & 1.064 & 1.062 \\
\hline & & & 14335 & & & & & & 1.137 & 1.132 & 1.128 & 1.124 \\
\hline & & & 1.14667 & & & & & 1.207 & 1.205 & 1.199 & 1.192 & 1.187 \\
\hline & & & & & & & & & & & & 1.249 \\
\hline & & & 1.15291 & 1.15075 & 1.14850 & 1.14594 & 1.372 & 1.357 & 1.342 & 1.330 & 1.320 & 1.311 \\
\hline & & & & -Alanina & $+\mathrm{V}\left[\mathrm{H}_{2} \mathrm{O}\right.$ & $\mathrm{Na}_{2} \mathrm{SO}_{4}$ & $6001 \mathrm{~m}$ & & & & & \\
\hline $\mathrm{T} / \mathrm{K}$ & 283.15 & 288.15 & 293.15 & 298.15 & 303.15 & 308.15 & 283.15 & 288.15 & 293.15 & 298.15 & 303.15 & 308.15 \\
\hline$m\left(\mathrm{~mol} \mathrm{Kg}^{-1}\right)$ & & & $\rho(g \cdot$ & $\left.m^{-3}\right)$ & & & & & $\pi$ & Ir & & \\
\hline 000 & & & & & & & 1.000 & 1.000 & 1.000 & 1.000 & 1.000 & 1.000 \\
\hline 303 & & 1.18060 & 1.17833 & & & & 1.014 & 1.013 & 1.013 & 1.012 & 1.012 & 1.011 \\
\hline & & & 1.17855 & & & & & & & & & \\
\hline & & & 1.17894 & & & & 1.037 & 1.036 & 1.034 & 1.033 & 1.032 & 1.030 \\
\hline 013 & & 1.18147 & 1.17920 & & & & 1.047 & 1.045 & 1.043 & & 1.040 & 1.038 \\
\hline & 3509 & 1.18292 & 1.18062 & & 1.17 & & 1.093 & 1.090 & 1.086 & & 1.079 & 1.075 \\
\hline & & & 1.18362 & & & & 1.184 & 1.179 & 1.170 & 1.164 & 1.156 & 1.149 \\
\hline & & & 1.18676 & & & & 1.276 & 1.269 & 1.256 & 1.246 & 1.235 & 1.224 \\
\hline 3050 & 19458 & 1.19237 & 1.19007 & 1.18765 & 1.18515 & & 1.365 & 1.360 & 1.342 & 1.329 & 1.314 & 1.300 \\
\hline 1.0001 & 1.19785 & 1.19561 & 1.19337 & 1.19088 & 1.18840 & 1.18589 & 1.459 & 1.446 & 1.425 & 1.409 & 1.390 & 1.372 \\
\hline
\end{tabular}

En la ecn. (1), $m$ es la molalidad $\left(\mathrm{mol} \cdot \mathrm{kg}^{-1}\right)$ de la DL- alanina en solución acuosa de $\mathrm{Na}_{2} \mathrm{SO}_{4}, \mathrm{M}$ es la masa molar del aminoácido $\left(\mathrm{g} \cdot \mathrm{mol}^{-1}\right), \rho_{0}$ y $\rho$ son las densidades $\left(\mathrm{g} \cdot \mathrm{cm}^{-3}\right)$ del solvente mixto o pseudobinario y de la solución, respectivamente. Los datos de los volúmenes molares aparentes $\left(V_{\varphi}\right)$ fueron ajustados por el método 
de los mínimos cuadrados y a partir de ellos se calcularon los volúmenes molares aparentes límites $\left(\mathrm{V}_{\varnothing}^{0}\right)$ usando la ecuación (2).

$$
V_{\varphi}=V_{\varphi}^{0}+S_{v} m
$$

Donde, $V_{\varnothing}^{0}$ es el volumen molar aparente límite que, por definición, está libre de interacciones soluto-soluto y, por lo tanto, proporciona una medida de las interacciones soluto-solvente y $S_{v}$ es una pendiente experimental y proporciona información con respecto a las interacciones soluto-soluto. Los valores de los volúmenes molares aparentes a dilución infinita $\left(V_{\varnothing}^{0}\right)$ y la pendiente experimental $\left(S_{v}\right)$ se presentan en la Tabla 2.

Tabla 2: Volumen molar aparente límite del soluto $\left(V_{\varnothing}^{0}\right)$, pendiente experimental $\left(\mathrm{S}_{\mathrm{v}}\right)$, Volumen molar aparente límite de transferencia $\left(\Delta_{\mathrm{tr}} \mathrm{V}_{\varnothing}^{0}\right)$, número de hidratación $\left(\mathrm{n}_{\mathrm{h}}\right)$ y segunda derivada del volumen molar aparente límite del soluto con temperatura $\left(\partial^{2} \mathrm{~V}_{\varnothing}^{0} / \partial \mathrm{T}^{2}\right)$ para la Alanina en solución acuosa de $\mathrm{Na}_{2} \mathrm{SO}_{4}$.

\begin{tabular}{|c|c|c|c|c|c|c|}
\hline $\begin{array}{l}\mathrm{T} / \mathrm{K}= \\
\text { DL-Alanina }+\mathrm{I}\left[\mathrm{H}_{2} \mathrm{O}+\mathrm{Na}\right.\end{array}$ & 283.15 & 288.15 & 293.15 & 298.15 & 303.15 & 308.15 \\
\hline \multicolumn{7}{|c|}{ DL-Alanina + I [ $\left.\mathrm{H}_{2} \mathrm{O}+\mathrm{Na}_{2} \mathrm{SO}_{4}(0.2002 \mathrm{~m})\right]$} \\
\hline $\mathrm{V}_{\varphi}^{0}\left(\mathrm{~cm}^{3} \cdot \mathrm{mol}^{-1}\right)$ & 60.82 & 61.07 & 61.20 & 61.41 & 61.57 & 61.8 \\
\hline $\mathrm{S}_{\mathrm{v}}\left(\mathrm{cm}^{3} \cdot \mathrm{kg}^{-1}\right)$ & 0.5932 & 0.6655 & 0.7329 & 0.7455 & 0.7822 & 0.7721 \\
\hline$\Delta_{t r} V_{\varnothing}^{0}\left(\mathrm{~cm}^{3} \cdot \mathrm{mol}^{-1}\right)$ & 1.75 & 1.45 & 1.24 & 1.16 & 1.01 & 0.88 \\
\hline $\begin{array}{l}\mathrm{n}_{\mathrm{h}} \\
\partial^{2} V_{\infty}^{0} / \partial T^{2}\left(\mathrm{~cm}^{3} \cdot \mathrm{mol}^{-1} \cdot \mathrm{K}^{-2}\right)\end{array}$ & 3.13 & 2.83 & $\begin{array}{l}2.61 \\
4.00\end{array}$ & $\begin{array}{l}2.38 \\
\mathrm{E}-05\end{array}$ & 2.14 & 1.87 \\
\hline \multicolumn{7}{|c|}{ DL-Alanina + II [ $\left.\mathrm{H}_{2} \mathrm{O}+\mathrm{Na}_{2} \mathrm{SO}_{4}(0.5998 \mathrm{~m})\right]$} \\
\hline $\mathrm{V}_{\varphi}^{0}\left(\mathrm{~cm}^{3} \cdot \mathrm{mol}^{-1}\right)$ & 62.25 & 62.45 & 62.68 & 62.85 & 63.05 & 63.29 \\
\hline $\mathrm{S}_{\mathrm{v}}\left(\mathrm{cm}^{3} \cdot \mathrm{kg}^{-1}\right)$ & 0.8656 & 0.8572 & 0.8324 & 0.8327 & 0.7541 & 0.6196 \\
\hline$\Delta_{t r} V_{\varnothing}^{0}\left(\mathrm{~cm}^{3} \cdot \mathrm{mol}^{-1}\right)$ & 3.18 & 2.83 & 2.72 & 2.6 & 2.49 & 2.37 \\
\hline $\mathrm{n}_{\mathrm{h}}$ & 2.60 & 2.35 & 2.13 & 1.95 & 1.73 & 1.50 \\
\hline$\partial^{2} V_{\varnothing}^{0} / \partial T^{2}\left(\mathrm{~cm}^{3} \cdot \mathrm{mol}^{-1} \cdot \mathrm{K}^{-2}\right)$ & \multicolumn{6}{|c|}{$7.00 \mathrm{E}-05$} \\
\hline \multicolumn{7}{|c|}{ DL-Alanina + III [ $\left.\mathrm{H}_{2} \mathrm{O}+\mathrm{Na}_{2} \mathrm{SO}_{4}(0.9005 \mathrm{~m})\right]$} \\
\hline $\mathrm{V}_{\varphi}^{0}\left(\mathrm{~cm}^{3} \cdot \mathrm{mol}^{-1}\right)$ & 63.25 & 63.43 & 63.70 & 63.99 & 64.20 & 64.45 \\
\hline $\mathrm{S}_{\mathrm{v}}\left(\mathrm{cm}^{3} \cdot \mathrm{kg}^{-1}\right)$ & 1.2813 & 1.2519 & 1.1774 & 1.0241 & 1.0408 & 0.8258 \\
\hline$\Delta_{t r} V_{\varnothing}^{0}\left(\mathrm{~cm}^{3} \cdot \mathrm{mol}^{-1}\right)$ & 4.18 & 3.81 & 3.74 & 3.74 & 3.64 & 3.53 \\
\hline $\mathrm{n}_{\mathrm{h}}$ & 2.23 & 2.01 & 1.80 & 1.60 & 1.41 & 1.21 \\
\hline$\partial^{2} V_{\varnothing}^{0} / \partial T^{2}\left(\mathrm{~cm}^{3} \cdot \mathrm{mol}^{-1} \cdot \mathrm{K}^{-2}\right)$ & \multicolumn{6}{|c|}{ 7.00E-05 } \\
\hline \multicolumn{7}{|c|}{ DL-Alanina + IV [ $\left.\mathrm{H}_{2} \mathrm{O}+\mathrm{Na}_{2} \mathrm{SO}_{4}(1.2000 \mathrm{~m})\right]$} \\
\hline $\mathrm{V}_{\varphi}^{0}\left(\mathrm{~cm}^{3} \cdot \mathrm{mol}^{-1}\right)$ & 64.10 & 64.22 & 64.35 & 64.48 & 64.59 & 64.76 \\
\hline $\mathrm{S}_{\mathrm{v}}\left(\mathrm{cm}^{3} \cdot \mathrm{kg}^{-1}\right)$ & 0.3327 & 0.3014 & 0.3050 & 0.2643 & 0.2375 & 0.2841 \\
\hline$\Delta_{t r} V_{\varnothing}^{0}\left(\mathrm{~cm}^{3} \cdot \mathrm{mol}^{-1}\right)$ & 5.03 & 4.60 & 4.39 & 4.23 & 4.03 & 3.84 \\
\hline & 1.92 & 1.74 & 1.59 & 1.45 & 1.30 & 1.13 \\
\hline$\partial^{2} V_{\varnothing}^{0} / \partial T^{2}\left(\mathrm{~cm}^{3} \cdot \mathrm{mol}^{-1} \cdot \mathrm{K}^{-2}\right)$ & \multicolumn{6}{|c|}{ 1.00E-04 } \\
\hline \multicolumn{7}{|c|}{ DL-Alanina + V $\left[\mathrm{H}_{2} \mathrm{O}+\mathrm{Na}_{2} \mathrm{SO}_{4}(1.6001 \mathrm{~m})\right]$} \\
\hline $\mathrm{V}_{\varphi}^{0}\left(\mathrm{~cm}^{3} \cdot \mathrm{mol}^{-1}\right)$ & 65.16 & 65.32 & 65.53 & 65.69 & 65.89 & 66.07 \\
\hline $\mathrm{S}_{\mathrm{v}}\left(\mathrm{cm}^{3} \cdot \mathrm{kg}^{-1}\right)$ & 1.8621 & 1.8758 & 1.9999 & 1.9596 & 2.0268 & 2.1147 \\
\hline$\Delta_{t r} V_{\varnothing}^{0}\left(\mathrm{~cm}^{3} \cdot \mathrm{mol}^{-1}\right)$ & 6.09 & 5.70 & 5.57 & 5.44 & 5.33 & 5.15 \\
\hline & 1.52 & 1.36 & 1.21 & 1.09 & 0.94 & 0.80 \\
\hline$\partial^{2} V_{\varnothing}^{0} / \partial T^{2}\left(\mathrm{~cm}^{3} \cdot \mathrm{mol}^{-1} \cdot \mathrm{K}^{-2}\right)$ & \multicolumn{6}{|c|}{$4.00 \mathrm{E}-05$} \\
\hline
\end{tabular}

Los valores de $V_{\varnothing}^{0}$ para el aminoácido aumentan con el aumento en la concentración de la solución acuosa de sulfato de sodio y con el incremento de la temperatura, al igual como se presenta en los estudios realizado por Rodríguez y otros 2017 y Kumar y otros 2017, tal como se puede apreciar en la Tabla 2. En esta tabla se observa que los valores de $\mathrm{V}_{\varnothing}^{0}$ son positivos y aumentan con la molalidad de la solución acuosa de $\mathrm{Na}_{2} \mathrm{SO}_{4}$; este comportamiento en el estado de dilución infinita y $\mathrm{pH}$ neutro, podría ser asociado con el favorecimiento de fuertes interacciones entre el zwitterion del aminoácido y los iones hidratados de la sal. Además, el aumento del $V_{\varnothing}^{0}$ con el incremento de la temperatura se podría atribuir a una reducción del efecto de electrostricción. Mientras que los valores pequeños y positivos para $S_{v}$ a cada temperatura podrían indicar la presencia en la solución de débiles interacciones soluto-soluto (Kumar y otros 2017); (Páez y Cantero 2012). 
El análisis del comportamiento del volumen molar aparente a dilución infinita con temperatura, se realizó usando el criterio de Hepler, (Páez y Pérez 2015) encontrándose que $\partial^{2} \mathrm{~V}_{\varnothing}^{0} / \partial \mathrm{T}^{2}$ (Tabla 2) resultó positiva para la DL-alanina en las mezclas pseudobinarias, lo que indica su efecto formador sobre la estructura del solvente. Estas características son similares a las observadas para algunos aminoácidos en soluciones acuosas de sales (Amalendu y otro 2012; Ren y otros 2016; Sinha y otros 2010).

Por otra parte, el volumen molar aparente límite de transferencia $\left(\Delta_{\mathrm{tr}} \mathrm{V}_{\varnothing}^{0}\right)$, de la DL-alanina desde agua a soluciones acuosas de sulfato de sodio a dilución infinita, determinado con la ecuación (3).

$$
\Delta_{\mathrm{tr}} \mathrm{V}_{\varphi}^{0}=\mathrm{V}_{\varphi}^{0}(\text { en Sulfatode Sodioacuoso })-\mathrm{V}_{\varphi}^{0}(\text { en Agua })
$$

Resultó positivo (Tabla 2) y se observa que aumenta al incrementarse la temperatura y la concentración de sulfato de $\mathrm{Na}_{2} \mathrm{SO}_{4}$. Un comportamiento similar también ha sido reportado en otros sistemas de aminoácidos en medios electrolíticos; (Karanth 2013; Páez y Cantero 2012). Estos resultados pueden explicarse con ayuda del modelo de coesferas solapadas, desarrollado por Gurney (Mishra 1984), en el que se sugiere que el solapamiento de las coesferas de hidratación del aminoácido con las coesferas de los iones de la sal, libera algunas moléculas de agua desde las esferas de hidratación ocasionando que aumente la estructura global del agua dando lugar a un cambio positivo en el $\Delta_{\mathrm{tr}} \mathrm{V}_{\varnothing}^{0}$.

El número de moléculas de agua $\left(\mathrm{n}_{h}\right)$ que hidratan al aminoácido en solución acuosa de sulfato de sodio o número de hidratación, se puede estimar a partir del volumen molar parcial de electrostricción a partir del modelo propuesto por Millero 1978, ecuación (4):

$$
n_{h}=\frac{V_{2}^{0} \text { elect }}{V_{e}^{0}-V_{b}^{0}}
$$

Dónde: $V_{2 e l e c t}^{0}$ es el volumen de electrostricción y está dado por $V_{2 \text { elect }}^{0}=V_{\varnothing}^{0}-V_{\text {int }}^{0}$, aquí $V_{\text {int }}^{0}$ es el volumen parcial intrínseco del aminoácido, el cual se expresa en términos, del volumen de Van der Waals, asociado con los huecos o espacios vacíos y del volumen de contracción debido al efecto de electrostricción (Páez y Vergara 2015). Para calcular el $V_{\text {int }}^{0}$ se usa la ecuación (5), planteada por Millero 1978.

$$
V_{\text {int }}^{0}=\left(\frac{0.7}{0.634}\right) V_{\text {cris }}^{0}
$$

Donde $\mathrm{V}_{\text {cris }}^{0}$ es el volumen del cristal y se obtiene dividiendo la masa molar del cristal $\left(\mathrm{Na}_{2} \mathrm{SO}_{4}\right)$ por su densidad. Tanto la densidad del sólido y el volumen del cristal se asume constante en todo el rango de temperatura de trabajo. Pese a que Millero utilizó esta ecuación para aminoacidos en agua, esta ha sido utilizada también en solventes acuosos mixtos (Anwar y Shahla 2007; Nain 2016; Wang y otros 2014). Donde 0.7 es la densidad de empaquetamiento para la molécula en un cristal orgánico y 0.634 es la densidad de empaquetamiento para las esferas de empaquetamiento al azar. Este modelo supone que para cada molécula de agua tomada desde la fase volumétrica a la región acuosa que rodea el soluto, el volumen se reduce en $\left(\mathrm{V}_{\mathrm{e}}^{0}-V_{b}^{0}\right)$. Usando los valores para $\left(\mathrm{V}_{\mathrm{e}}^{0}-V_{b}^{0}\right)$ de $-2.9,-3.3,-4.0 \mathrm{~cm}^{3} \cdot \mathrm{mol}^{-1}$ a $288.15,298.15$ y $308.15 \mathrm{~K}$ respectivamente, y aquellos reportados por Wang y otros 2004 y McMillan y Mayer 1945 desde 278.15 hasta $313.15 \mathrm{~K}$; es posible usar la interpolación gráfica para obtener los valores de: $-2.7,-3.1,-3.6$ y $-4.6 \mathrm{~cm}^{3} \cdot \mathrm{mol}^{-}$ ${ }^{1}$ para temperaturas $283.15,293.15,303.15$ y $313.15 \mathrm{~K}$.

Los números de hidratación $\left(\mathrm{n}_{h}\right)$ calculados en este trabajo para la DL-alanina en solución acuosa de $\mathrm{Na}_{2} \mathrm{SO}_{4}$ se reportan en la Tabla 2. Puede observarse que los números de hidratación $\left(\mathrm{n}_{\mathrm{h}}\right)$ de la DL-alanina en solución acuosa de $\mathrm{Na}_{2} \mathrm{SO}_{4}$ resultan positivos y en general decrecen al aumentar la temperatura y la concentración de la sal en el solvente mixto. Este último comportamiento se puede atribuir a un incremento de la interacción entre las partes zwitterionicas del aminoácido y los iones de la sal, lo que conduce a una reducción del efecto de electrostricción en las moléculas de agua (Páez y Cantero 2012). Este análisis es consistente con el expuesto para los volúmenes de transferencia. Los datos de viscosidad relativa $\left(\eta_{r}\right)$ a las temperaturas de 283.15 a $308.15 \mathrm{~K}$ obtenidos para cada una de las mezclas pseudobinarias DL-alanina $+\mathrm{Na}_{2} \mathrm{SO}_{4}$ acuoso, se muestran en la Tabla 1, en función de la molalidad $\left(\mathrm{mol} \cdot \mathrm{kg}^{-1}\right)$ del aminoácido. El análisis del comportamiento de los datos de $\eta_{r}$ con concentración, se realizó usando la ecuación (6) de Jones y Dole 1929, de acuerdo con la cual:

$$
\eta_{r}=\frac{\eta}{\eta_{0}}=1+A C^{1 / 2}+B C+D C^{2}
$$


Donde $(\eta)$ es la viscosidad dinámica de la solución, ( $\left.\eta_{0}\right)$ es la viscosidad del disolvente pseudobinario (agua + $\left.\mathrm{Na}_{2} \mathrm{SO}_{4}\right), C\left(\mathrm{~mol}^{\circ} \mathrm{L}^{-1}\right)$ es la molaridad de la DL-alanina en la solución, y A, B y D son parámetros ajustables. Vale la pena aclarar que A (coeficiente de Falkenhagen) es una constante siempre positiva, que mide las interacciones de largo alcance ion-ion (Páez y Vergara 2015); B es un parámetro empírico de viscosidad, que tiene en cuenta la interacción ion-solvente; mientras que el coeficiente $D$ incluye todas las interacciones estructurales soluto-solvente y soluto-soluto que no se toman en cuenta en los términos $\mathrm{AC}^{1 / 2}$ y BC (Jones y Dole 1929; Páez y Alvis 2014). Los resultados de la correlación dada por la ecuación (6), se muestran en la Tabla 3.

Tabla 3: Coeficientes de Viscosidad A, B y D de la ecuación de Jones-Dole, para la mezcla DL-alanina + solución acuosas de $\mathrm{Na}_{2} \mathrm{SO}_{4}$ a diferentes temperaturas.

\begin{tabular}{|c|c|c|c|c|c|c|}
\hline & $\mathrm{T}=283.15$ & & $T=293.15$ & & 3.15 & \\
\hline \multicolumn{7}{|c|}{ DL-Alanina + I [ $\left.\mathrm{H}_{2} \mathrm{O}+\mathrm{Na}_{2} \mathrm{SO}_{4}(0.2002 \mathrm{~m})\right]$} \\
\hline$A\left(L^{1 / 2} \cdot \mathrm{mol}^{-1 / 2}\right)$ & $9.768 \mathrm{E}-04$ & $1.087 \mathrm{E}-03$ & $1.219 \mathrm{E}-03$ & $1.344 \mathrm{E}-03$ & 1.471 & $1.470 \mathrm{E}-03$ \\
\hline $\mathrm{B}\left(\mathrm{L} \cdot \mathrm{mol}^{-1}\right)$ & 0.32584 & 0.30719 & 0.29787 & 0.28338 & 0.25895 & 0.25495 \\
\hline$D\left(L^{2} \cdot r\right.$ & -0.00627 & -0.00587 & -0.00577 & -0.00482 & -0.01171 & 0.00061 \\
\hline \multicolumn{7}{|c|}{ DL-Alanina $+\mathrm{II}\left[\mathrm{H}_{2} \mathrm{O}+\mathrm{Na}_{2} \mathrm{SO}_{4}(0.5998 \mathrm{~m})\right]$} \\
\hline$A\left(L^{1 / 2} \cdot \mathrm{mol}^{-1 / 2}\right)$ & $8.501 \mathrm{E}-04$ & $9.261 \mathrm{E}-04$ & $1.025 \mathrm{E}-03$ & $1.131 \mathrm{E}-03$ & $1.245 \mathrm{E}-03$ & 1.367E-03 \\
\hline $\mathrm{B}\left(\mathrm{L} \cdot \mathrm{mol}^{-1}\right)$ & 0.35135 & 0.33828 & 0.32654 & 0.31111 & 0.30452 & 0.29339 \\
\hline $\mathrm{D}\left(\mathrm{L}^{2} \cdot \mathrm{mol}^{-2}\right)$ & -0.00699 & -0.00647 & -0.00615 & -0.00576 & 0586 & -0.00489 \\
\hline \multicolumn{7}{|c|}{ DL-Alanina + III [ $\left[\mathrm{H}_{2} \mathrm{O}+\mathrm{Na}_{2} \mathrm{SO}_{4}(0.9005 \mathrm{~m})\right]$} \\
\hline $\mathrm{A}\left(\mathrm{L}^{1 / 2} \cdot \mathrm{mol}^{-1 / 2}\right)$ & 7.637E-04 & 8.193E-04 & 8.905E-04 & $9.867 \mathrm{E}-04$ & $1.089 \mathrm{E}-03$ & 1.204E-03 \\
\hline $\mathrm{B}\left(\mathrm{L} \cdot \mathrm{mol}^{-1}\right)$ & 0.36160 & 0.34756 & 0.33479 & 0.32059 & 0.31057 & 0.30229 \\
\hline $\mathrm{D}\left(\mathrm{L}^{2} \cdot \mathrm{mol}^{-2}\right)$ & -0.00734 & -0.00670 & -0.00677 & -0.00565 & -0.00658 & -0.00669 \\
\hline \multicolumn{7}{|c|}{ DL-Alanina + IV $\left[\mathrm{H}_{2} \mathrm{O}+\mathrm{Na}_{2} \mathrm{SO}_{4}(1.2000 \mathrm{~m})\right]$} \\
\hline$A\left(L^{1 / 2} \cdot \mathrm{mol}^{-1 / 2}\right)$ & $6.838 \mathrm{E}-04$ & $7.262 \mathrm{E}-04$ & 7.902E-04 & 8.597E-04 & 9.487E-04 & 1.057E-03 \\
\hline $\mathrm{B}\left(\mathrm{L} \cdot \mathrm{mol}^{-1}\right)$ & 0.34770 & 0.33236 & 0.31959 & 0.30822 & 0.29831 & 0.29007 \\
\hline$\left(\mathrm{L}^{2} \cdot \mathrm{mol}^{-2}\right)$ & -0.00645 & -0.01679 & -0.00649 & -0.00591 & -0.00581 & -0.00593 \\
\hline \multicolumn{7}{|c|}{ DL-Alanina + V $\left[\mathrm{H}_{2} \mathrm{O}+\mathrm{Na}_{2} \mathrm{SO}_{4}(1.6001 \mathrm{~m})\right]$} \\
\hline$A\left(L^{1 / 2} \cdot \mathrm{mol}^{-1 / 2}\right)$ & $6.031 \mathrm{E}-04$ & $6.354 \mathrm{E}-04$ & $6.801 \mathrm{E}-04$ & 7.337E-04 & 8.039E-04 & 8.971E-04 \\
\hline $\mathrm{B}\left(\mathrm{L} \cdot \mathrm{mol}^{-1}\right)$ & 0.39236 & 0.37672 & 0.35884 & 0.34421 & 0.32801 & 0.31316 \\
\hline $\mathrm{D}\left(\mathrm{L}^{2} \cdot \mathrm{mol}^{-2}\right)$ & 0.01637 & 0.02625 & 0.02414 & 0.02436 & 0.02359 & 0.02225 \\
\hline
\end{tabular}

Puede observarse que el coeficiente $A$ resulta con valores pequeños y positivos, típicos de los sistemas electrolíticos (Jones y Dole 1929; Páez y Vergara 2015), no obstante este valor se ve opacado por los valores del coeficiente $B$, que resultan ser positivos y mucho más grandes, para la DL-alanina en todos los solventes pseudobinarios y a todas las temperaturas de estudio, lo que sugiere que en estos sistemas predomina la interacción entre las partes zwitterionicas del aminoácido y los iones de la sal (Chunyan y otros 2018; Xiaofeng y Chunying 2014), este resultado es consistente con los análisis previos del número de hidratación. En adición, $\partial \mathrm{B} / \partial \mathrm{T}$, resultó negativo indicando que la $\mathrm{DL}$-alanina en la región diluida, se comporta como un soluto formador de la estructura del solvente, tal como se ilustra en la Figura 1. Mientras, que el valor del coeficiente $\mathrm{D}$ distinto de cero podría estar indicar la presencia de autointeracciones iónicas e interacciones entre iones de signos opuestos (Xiaofeng y Chunying 2014).

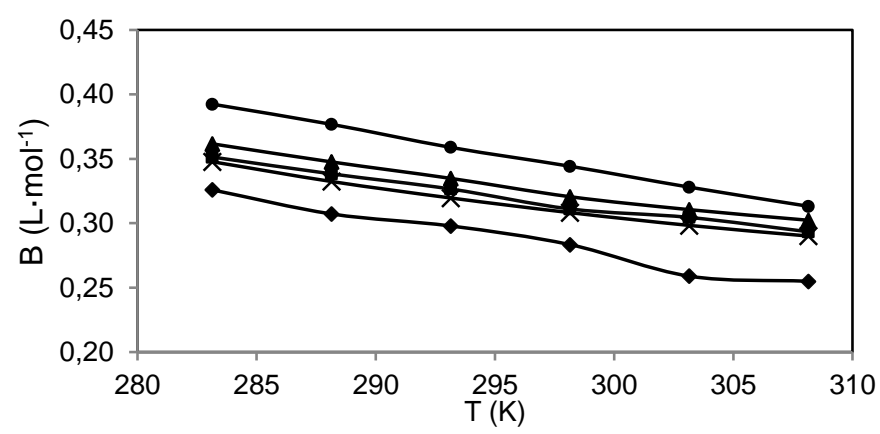

Fig.1: Variación del coeficiente $\mathrm{B}$ de viscosidad con temperatura $(\partial \mathrm{B} / \partial \mathrm{T})$ para el aminoácido DL-Alanina en soluciones acuosas de $\mathrm{Na}_{2} \mathrm{SO}_{4}$ : (I) $\bullet 0.2000 \mathrm{~m}$ $\left(\mathrm{H}_{2} \mathrm{O}+\mathrm{Na}_{2} \mathrm{SO}_{4}\right)$, (II) $\times 0.5998 m\left(\mathrm{H}_{2} \mathrm{O}+\mathrm{Na}_{2} \mathrm{SO}_{4}\right)$, (III) $=0.9005 m\left(\mathrm{H}_{2} \mathrm{O}+\right.$ $\left.\mathrm{Na}_{2} \mathrm{SO}_{4}\right),\left(\right.$ IV) $\Delta 1.2000 m\left(\mathrm{H}_{2} \mathrm{O}+\mathrm{Na}_{2} \mathrm{SO}_{4}\right)$ y $(\mathbf{V}) \bullet 1.6001 m\left(\mathrm{H}_{2} \mathrm{O}+\mathrm{Na}_{2} \mathrm{SO}_{4}\right)$. 
Finalmente, el análisis de los parámetros termodinámicos de activación del flujo viscoso, se realizó con ayuda de la teoría de las velocidades absoluta de Erying (Páez y Alvis 2014; Feakins y otros 1974; Glasstone y otros 1941), mediante un procedimiento de regresión lineal, usando las ecuaciones (7) y (8).

$$
\begin{aligned}
& L n \frac{\eta V_{m}}{h N}=\frac{\Delta H^{\neq}}{R T}-\frac{\Delta S^{\neq}}{R} \\
& \Delta G^{\neq}=\Delta H^{\neq}-T \Delta S^{\neq}
\end{aligned}
$$

Donde $\mathrm{h}$ es la constante de Planck, $\mathrm{N}$ es el número de Avogadro, $\mathrm{V}_{\mathrm{m}}$ es el volumen molar del sistema, $\mathrm{T}$ es la temperatura absoluta, y $\Delta \mathrm{G}^{\neq}, \Delta \mathrm{H}^{\neq}$y $\Delta \mathrm{S}^{\neq}$son la energía libre de Gibbs, la entalpía y la entropía de activación del flujo viscoso respectivamente (Feakins y otros 1974). Los resultados de la correlación grafica del $\operatorname{Ln}\left(\eta V_{m} / h N\right)$ vs $1 / T$ para los parámetros termodinámicos de activación del flujo viscoso, respecto a cada punto de composición del sistema DL-alanina + solución acuosa de $\mathrm{Na}_{2} \mathrm{SO}_{4}$, se muestran en la Tabla 4. La energía libre de activación a luz de teoría de Eyring, (Glasstone y otros 1941), es entendida como el exceso de energía libre que posee una molécula localizada en la cercanía de una cavidad de un sistema líquido para poder saltar hacia esa cavidad durante el proceso de flujo viscoso. Aquí las interacciones moleculares entre las especies en el sistema juegan un papel clave, ya que si estas son fuertes la velocidad del flujo viscoso disminuye y la viscosidad aumenta y viceversa.

En adición, un signo positivo para $\Delta \mathrm{G}^{\star}$ evidencia una dificultad mayor en el movimiento de las moléculas de soluto para desplazarse a través del disolvente, de ahí que se necesite mayor energía para saltar de una posición de equilibrio a otra, y en consecuencia la viscosidad aumenta. Este hecho, junto a un $\Delta \mathrm{H}^{\neq}$positivo (proceso endotérmico), indica la desfavorabilidad termodinámica del proceso, por cuanto es necesario suministrar energía para superar la barrera energética del proceso de flujo viscoso. Además, la disminución de la energía libre de activación $\left(\Delta G^{*}\right)$ con la temperatura indica que la barrera de energía para la formación de cavidades es más pequeña, lo que aumenta la velocidad del flujo viscoso. En particular el sistema DLalanina + solución acuosa de sulfato de sodio, tal como se observa en la Tabla 4, presentó valores positivos para $\Delta \mathrm{G}^{\neq} \mathrm{y} \Delta \mathrm{H}^{\neq} \mathrm{y}$ valores negativos para $\Delta \mathrm{S}^{\neq}$. No obstante, el hecho de que $\Delta \mathrm{S}^{\neq}$para el sistema aquí estudiado, sea negativo implica que el complejo activado resulta más ordenado, respecto al estado inicial, aunque el costo energético sea mayor (Sinha y Sarkar 2011; Khanuja 2013).

En términos generales puede decirse que los valores negativos de $\Delta S^{\neq}$a todas las temperaturas $y$ composiciones del solvente mixto, sugieren que la formación del estado de transición va acompañada de una formación de enlaces intermoleculares y una estructura más ordenada de las especies en el estado activado. Este comportamiento se ha observado en otros sistemas de aminoácidos con solventes mixtos (Biswajit y otros 2010; Sinha y otros 2010)

Tabla 4: Parámetros de activación del flujo viscoso para DL-alanina + solvente Pseudobinario

\begin{tabular}{|c|c|c|c|c|c|c|c|c|}
\hline & $\mathrm{T} / \mathrm{K}$ & & 283.15 & 288.15 & 293.15 & 298.15 & 303.15 & 308.15 \\
\hline$m_{a a}$ & $\Delta \mathrm{H}^{z}$ & $\Delta S^{\neq}$ & \multicolumn{6}{|c|}{$\Delta \mathrm{G}^{ \pm}$} \\
\hline $\mathrm{Kg} \cdot \mathrm{mol}^{-1}$ & $\mathrm{~kJ} \cdot \mathrm{mol}^{-1}$ & $\mathrm{~J} \cdot \mathrm{mol}^{-1} \mathrm{~K}^{-1}$ & \multicolumn{6}{|c|}{$\mathrm{kJ} \cdot \mathrm{mol}^{-1}$} \\
\hline \multicolumn{9}{|c|}{ DL-Alanina + I $\left[\mathrm{H}_{2} \mathrm{O}+\mathrm{Na}_{2} \mathrm{SO}_{4}(0.2002 \mathrm{~m})\right]$} \\
\hline 0.0000 & 16.72 & -24.61 & 9.75 & 9.63 & 9.51 & 9.38 & 9.26 & 9.14 \\
\hline 0.0306 & 16.82 & -24.94 & 9.76 & 9.64 & 9.51 & 9.39 & 9.26 & 9.14 \\
\hline 0.0509 & 16.91 & -25.13 & 9.79 & 9.67 & 9.54 & 9.42 & 9.29 & 9.16 \\
\hline 0.0803 & 16.97 & -25.27 & 9.81 & 9.69 & 9.56 & 9.43 & 9.31 & 9.18 \\
\hline 0.1010 & 17.07 & -25.58 & 9.83 & 9.70 & 9.58 & 9.45 & 9.32 & 9.19 \\
\hline 0.1967 & 17.40 & -26.48 & 9.90 & 9.77 & 9.64 & 9.51 & 9.37 & 9.24 \\
\hline 0.4027 & 18.03 & -28.19 & 10.05 & 9.91 & 9.76 & 9.62 & 9.48 & 9.34 \\
\hline 0.6014 & 18.63 & -29.82 & 10.18 & 10.03 & 9.88 & 9.73 & 9.58 & 9.44 \\
\hline 0.8023 & 19.17 & -31.29 & 10.31 & 10.15 & 10.00 & 9.84 & 9.68 & 9.53 \\
\hline 1.0006 & 19.73 & -32.85 & 10.43 & 10.27 & 10.10 & 9.94 & 9.77 & 9.61 \\
\hline \multicolumn{9}{|c|}{ DL-Alanina $+\mathrm{II}\left[\mathrm{H}_{2} \mathrm{O}+\mathrm{Na}_{2} \mathrm{SO}_{4}(0.5998 \mathrm{~m})\right]$} \\
\hline 0.0000 & 15.99 & -20.77 & 10.11 & 10.00 & 9.90 & 9.80 & 9.69 & 9.59 \\
\hline 0.0318 & 16.08 & -21.01 & 10.14 & 10.03 & 9.93 & 9.82 & 9.72 & 9.61 \\
\hline 0.0507 & 16.15 & -21.18 & 10.15 & 10.05 & 9.94 & 9.84 & 9.73 & 9.62 \\
\hline 0.0791 & 16.24 & -21.41 & 10.18 & 10.07 & 9.96 & 9.86 & 9.75 & 9.64 \\
\hline 0.0997 & 16.29 & -21.49 & 10.20 & 10.09 & 9.99 & 9.88 & 9.77 & 9.66 \\
\hline 0.2005 & 16.59 & -22.28 & 10.28 & 10.17 & 10.06 & 9.94 & 9.83 & 9.72 \\
\hline 0.4000 & 17.17 & -23.79 & 10.44 & 10.32 & 10.20 & 10.08 & 9.96 & 9.84 \\
\hline 0.6003 & 17.60 & -24.78 & 10.59 & 10.46 & 10.34 & 10.21 & 10.09 & 9.97 \\
\hline 0.7999 & 18.10 & -26.05 & 10.73 & 10.60 & 10.47 & 10.34 & 10.21 & 10.08 \\
\hline 0.9985 & 18.58 & -27.25 & 10.86 & 10.73 & 10.59 & 10.45 & 10.32 & 10.18 \\
\hline
\end{tabular}
a diferentes concentraciones del aminoácido y diferentes temperaturas. 
Tabla 4 (continuación)

\begin{tabular}{|c|c|c|c|c|c|c|c|c|}
\hline \multicolumn{9}{|c|}{ DL-Alanina + III $\left[\mathrm{H}_{2} \mathrm{O}+\mathrm{Na}_{2} \mathrm{SO}_{4}(0.9005 \mathrm{~m})\right]$} \\
\hline 0.0000 & 15.43 & -17.79 & 10.39 & 10.30 & 10.21 & 10.12 & 10.03 & 9.94 \\
\hline 0.0313 & 15.53 & -18.04 & 10.42 & 10.33 & 10.24 & 10.15 & 10.06 & 9.97 \\
\hline 0.0506 & 15.59 & -18.19 & 10.44 & 10.34 & 10.25 & 10.16 & 10.07 & 9.98 \\
\hline 0.0808 & 15.69 & -18.47 & 10.46 & 10.37 & 10.28 & 10.19 & 10.09 & 10.00 \\
\hline 0.1000 & 15.75 & -18.62 & 10.48 & 10.39 & 10.29 & 10.20 & 10.11 & 10.02 \\
\hline 0.2005 & 16.06 & -19.38 & 10.57 & 10.47 & 10.38 & 10.28 & 10.18 & 10.09 \\
\hline 0.4001 & 16.63 & -20.81 & 10.74 & 10.63 & 10.53 & 10.42 & 10.32 & 10.22 \\
\hline 0.6003 & 17.10 & -21.92 & 10.89 & 10.79 & 10.68 & 10.57 & 10.46 & 10.35 \\
\hline 0.8000 & 17.61 & -23.19 & 11.04 & 10.93 & 10.81 & 10.70 & 10.58 & 10.46 \\
\hline 1.0001 & 18.08 & -24.35 & 11.19 & 11.06 & 10.94 & 10.82 & 10.70 & 10.58 \\
\hline \multicolumn{9}{|c|}{ DL-Alanina $+\mathrm{IV}\left[\mathrm{H}_{2} \mathrm{O}+\mathrm{Na}_{2} \mathrm{SO}_{4}(1.2000 \mathrm{~m})\right]$} \\
\hline 0.0000 & 14.70 & -14.24 & 10.66 & 10.59 & 10.52 & 10.45 & 10.38 & 10.31 \\
\hline 0.0310 & 14.80 & -14.77 & 10.62 & 10.54 & 10.47 & 10.40 & 10.32 & 10.25 \\
\hline 0.0507 & 14.85 & -14.89 & 10.63 & 10.56 & 10.49 & 10.41 & 10.34 & 10.26 \\
\hline 0.0816 & 14.93 & -15.07 & 10.66 & 10.58 & 10.51 & 10.43 & 10.36 & 10.28 \\
\hline 0.1002 & 14.99 & -15.23 & 10.68 & 10.60 & 10.52 & 10.45 & 10.37 & 10.30 \\
\hline 0.2000 & 15.30 & -16.01 & 10.77 & 10.69 & 10.61 & 10.52 & 10.44 & 10.36 \\
\hline 0.3993 & 15.86 & -17.39 & 10.93 & 10.84 & 10.76 & 10.67 & 10.58 & 10.50 \\
\hline 0.6008 & 16.31 & -18.44 & 11.09 & 10.99 & 10.90 & 10.81 & 10.72 & 10.63 \\
\hline 0.8000 & 16.84 & -19.78 & 11.24 & 11.14 & 11.04 & 10.94 & 10.84 & 10.74 \\
\hline 1.0001 & 17.38 & -21.19 & 11.38 & 11.28 & 11.17 & 11.06 & 10.96 & 10.85 \\
\hline \multicolumn{9}{|c|}{ DL-Alanina $+\mathrm{V}\left[\mathrm{H}_{2} \mathrm{O}+\mathrm{Na}_{2} \mathrm{SO}_{4}(1.6001 \mathrm{~m})\right]$} \\
\hline 0.0000 & 13.44 & -8.72 & 10.97 & 10.93 & 10.89 & 10.84 & 10.80 & 10.76 \\
\hline 0.0303 & 13.56 & -9.62 & 10.84 & 10.79 & 10.74 & 10.69 & 10.64 & 10.60 \\
\hline 0.0502 & 13.64 & -9.80 & 10.86 & 10.81 & 10.76 & 10.71 & 10.66 & 10.61 \\
\hline 0.0809 & 13.76 & -10.13 & 10.89 & 10.84 & 10.79 & 10.74 & 10.69 & 10.64 \\
\hline 0.1013 & 13.84 & -10.31 & 10.92 & 10.87 & 10.81 & 10.76 & 10.71 & 10.66 \\
\hline 0.2014 & 14.23 & -11.30 & 11.03 & 10.97 & 10.91 & 10.86 & 10.80 & 10.74 \\
\hline 0.3999 & 14.93 & -13.09 & 11.23 & 11.16 & 11.10 & 11.03 & 10.97 & 10.90 \\
\hline 0.6013 & 15.51 & -14.46 & 11.42 & 11.34 & 11.27 & 11.20 & 11.13 & 11.06 \\
\hline 0.8050 & 16.06 & -15.76 & 11.59 & 11.51 & 11.43 & 11.35 & 11.28 & 11.20 \\
\hline 1.0001 & 16.66 & -17.32 & 11.76 & 11.67 & 11.67 & 11.67 & 11.67 & 11.67 \\
\hline
\end{tabular}

\section{CONCLUSIONES}

En este estudio se reportan datos volumétricos, viscosimétricos y termodinámicos para la DL-alanina en soluciones acuosas de sulfato de sodio a diferentes temperaturas. El análisis del comportamiento del número de hidratación con la temperatura y la concentración de la sal, sugiere que en este sistema existe un favorecimiento de las interacciones: zwitterion hidratado del aminoácido e iones hidratados de la sal, con una reducción del efecto de electrostricción de las moléculas de agua. Además los valores positivos de $\partial^{2} V_{\varnothing}^{0} / \partial \mathrm{T}^{2}$ y negativos de $\partial \mathrm{B} / \partial \mathrm{T}$, evidencian que la $\mathrm{DL}$-alanina se comporta como un soluto formador de la estructura del solvente, con un ligero favorecimiento de la estructura global del agua, tal como se infiere del análisis del comportamiento del volumen de transferencia. También se encontró que los parámetros de activación del flujo viscoso para el sistema DL-alanina + solución acuosa de sulfato de sodio, resultaron positivos para $\Delta \mathrm{G}^{*}$ y $\Delta \mathrm{H}^{\neq}$y negativos para $\Delta \mathrm{S}^{\neq}$. Este último hecho sugiere, que la formación del estado de transición va acompañado de una formación de enlaces intermoleculares y una estructura más ordenada de las especies en el estado activado.

\section{AGRADECIMIENTOS}

Los autores agradecen a la U. de Córdoba por el apoyo prestado para la realización de este trabajo, en el laboratorio de Termodinámica de Soluciones del Departamento de Química, Facultad de Ciencias Básicas.

\section{REFERENCIAS}

Amalendu, P. y S. Surbhi, Volumetric Properties of Glycine in Aqueous Solutions of Some Sulfa Drugs at (288.15, 298.15, and 308.15) K, doi:10.1021/je300455e, J. Chem. Eng. Data, 58, 18-23 (2012)

Anwar, A., K. Shahla, H. Soghra y T. Mohd, Interactions of Some a-Amino acids with Tetra-n-Alkylammonium Bromides in Aqueous medium at Different Temperatures, doi:10.1016/j.jct.2006.08.010, J. Chem. Thermodyn., 39(4), 613-620 (2007)

Biswajit, S., K.R. Pran y N.R. Mahendra, Apparent Molar Volumes and Viscosity B-Coefficients of Glycine in Aqueous Silver Sulphate Solutions at $T=(298.15,308.15,318.15)$ K., ISSN: 1580-3155, Acta Chim. Slov., 57, $651-659$ (2010)

Chunyan, C., Z. Chunying, F. Taotao y M. Youguang, Volumetric and Viscometric Properties of Aqueous Solutions of Sodium amino Acids at $\mathrm{T}=(293.15$ to 333.15) K., doi: 10.1016/j.molliq.2018.01.058, J. Mol. Liq., 253, 241-249 (2018) 
Feakins, D., D.J. Freemantle y K.G. Lawrence, Transition state treatment of the relative viscosity of electrolytic solutions. Applications to aqueous, non-aqueous and methanol + water systems, doi: 10.1039/F19747000795, J. Chem. Soc., Faraday Trans., 70, 795-806 (1974)

Glasstone, S., K. Laidler, H. Eyring, The Theory of Rate Processes, McGraw-hill, New York, 477 (1941)

Jones, G. y M. Dole, The Viscosity of Aqueous Solutions of Strong electrolytes with Special reference to Barium Chloride, doi: 10.1021/ja01385a012, J. Am. Chem. Soc., 51(10), 2950-2964 (1929)

Karanth, V.R. y D.K. Bhat, Partial molar Volume and Partial molar Isentropic Compressibility Study of Glycine betaine in Aqueous and Aqueous $\mathrm{KCl}$ or $\mathrm{MgCl}_{2}$ Solutions at Temperatures $\mathrm{T}=288.15-318.15 \mathrm{~K}$, doi:10.1016/j.tca.2013.08.002, Thermochim. Acta, 572, 23-29 (2013)

Khanuja, P., Volumetric and Viscometric Study of interactions of Amino acids in Aqueous sucrose Solution at different Temperaturas, doi:10.7598/cst2013.524, Chem. Sci. Trans., 2(4), 1268-1275 (2013)

Kumar, D., S.K. Lomesh y V. Nathan, Molecular Interaction Studies of L-alanine and L-Phenylalanine in Water and in Aqueous Citric Acid at Different Temperatures using Volumetric, Viscosity and Ultrasonic Methods, doi:10.1016/j.molliq.2017.08.057, J. Mol. Liq., 247, 75-83 (2017)

Liu, C. y C. Ren, Transfer Properties of Amino Acids from Water to Aqueous Sodium Sulfate Solutions at $298.15 \mathrm{~K}$, doi:10.1021/je900218z, J. Chem. Eng. Data, 54(12), 3296-3299 (2009)

McMillan, W.G. y J.E. Mayer, The Statistical Thermodynamics of Multicomponent Systems, doi:10.1063/1.1724036, J. Chem. Phys., 13, 276-305 (1945)

Millero, F.J., A. Lo Surdo y C. Shin, The Apparent molal Volumes and Adiabatic Compressibilities of Aqueous Amino acids at $25^{\circ} \mathrm{C}$, doi: 10.1021/j100496a007, J. Phys. Chem., 82(7), 784-792 (1978)

Mishra, A.K. y J.C. Ahluwalia, Apparent molal Volumes of Amino acids, N-acetylamino acids, and Peptides in Aqueous Solutions, doi: 10.1021/j150645a021, J. Phys. Chem., 88(1), 86-92 (1984)

Nain, A.K., R. Pal y P. Droliya, Study of (solute + solute) and (solute + solvent) Interactions of Homologous series of Some a-Amino acids in Aqueous-Streptomycin Sulfate solutions at Different Temperatures by Using Physicochemical Methods, doi:10.1016/j.jct.2015.11.015, J. Chem. Thermodyn., 95, 77-98 (2016)

Páez, M.S., D.E. Pérez y O.A. Pérez, Propiedades Volumétricas de las Mezclas Binarias del Líquido lónico $[\mathrm{Bmim}]\left[\mathrm{CF}_{3} \mathrm{SO}_{3}\right]$ con Etanol, con 1-propanol y con Agua a diferentes Temperaturas, doi:10.4067/S071807642015000400014, Inf. Tecnol., 26(4), 105-114 (2015)

Páez, M.S., A. Alvis y G. Arrázola, Efecto de la Temperatura sobre la Viscosidad de Soluciones Acuosas diluidas de Cloruro de 1-Butil-3-metil Imidazolio [Bmim+][Cl], doi:10.4067/S0718-07642014000300021, Inf. Tecnol., 25(3), 185-190 (2014)

Páez, M.S., P.D. Cantero y E.J. Marzola, Densidades y Propiedades Volumétricas de la Glicina en Soluciones Acuosas de Tiosulfato de sodio Pentahidratado $\left(\mathrm{Na}_{2} \mathrm{~S}_{2} \mathrm{O}_{3} \cdot 5 \mathrm{H}_{2} \mathrm{O}\right)$ a Diferentes Temperaturas, ISSN electrónico 2357-3791, Rev. Colomb. Química, 41, 449-484 (2012)

Páez, M.S., M.K. Vergara y N.A. De la Espriella, Interacciones de la DL-Alanina en Soluciones Acuosas de Trifluorometanosulfonato de 1-Butil-3-Metilimidazolio a Diferentes Temperaturas, doi:10.4067/S0718-07642015000100007, Inf. Tecnol., 26, 63-70 (2015)

Partha, S.G., Kalachand, Mahali, K.D. Bijoy y R. Sanjay, Solubility and Thermodynamics of Solute-Solvent Interactions of Some Amino Acids in Aqueous Sodium Bromide and Potassium Bromide Solutions, doi: 10.1021/acs.jced.7b00647, J. Chem. Eng. Data, 63 (3), pp 534-541 (2018)

Pica, A. y G. Graziano, On the Effect of Sodium Chloride and Sodium Sulfate on Cold Denaturation, doi:10.1371/journal.pone.0133550, PLoS ONE, 10 (7), 1 - 13 (2015)

Rajagopal, K. y S.S. Jayabalakrishnan, Volumetric and Viscometric Studies of 4-Aminobutyric Acid in Aqueous Solutions of Salbutamol Sulphate at 308.15, 313.15 and 318.15 K, doi: 10.1016/S1004-9541(08)60279-7, Chinese J. Chem. Eng., 17(5), 796-804 (2009)

Ramasami, P., Solubilities of Amino Acids in Water and Aqueous Sodium Sulfate and Related Apparent Transfer Properties, doi:10.1021/je025503u, J. Chem. Eng. Data, 47(5), 1164-1166 (2002)

Ren, X., Z. Chunying y M. Youguang, Volumetric and Viscometric Study of Amino acids in Aqueous Sorbitol Solution at different Temperatures, doi:10.1016/j.jct.2015.10.002, J. Chem. Thermodyn., 93, 179-192 (2016)

Riddick, J.A. y W.B. Bunger, Organic Solvents, (Techniques of Chemistry), Third Edition, Wiley, 605 Third Ave., New York (1971)

Rodríguez, D.M., A.C. Ribeiro, M.A. Esteso y C.M. Romero, Effect of Temperature on the Partial molar Volumes, Partial molar Compressibility and Viscosity B-coefficients of DL-4-Aminobutyric acid in Water and in Aqueous Sodium Chloride Solutions, doi:10.1016/j.jct.2017.07.037, J. Chem. Thermodyn., 115, 98-105 (2017)

Romero, C.M., D.M. Rodríguez, A.C. Ribeiro y M.A. Esteso, Effect of Temperature on the Partial molar Volume, Isentropic Compressibility and Viscosity of DL-2-aminobutyric acid in Water and in Aqueous Sodium Chloride Solutions, doi:10.1016/j.jct.2016.09.040, J. Chem. Thermodyn., 104, 274-280 (2017) 
Sinha, B., P.K. Roy y otros 3 autores, Apparent molar Volumes and Viscosity B-coefficients of Glycine in Aqueous Silver sulphate Solutions at $T=(298.15,308.15,318.15)$ K., doi:10.1016/j.jct.2009.09.010, Acta Chim. Slov., 42, 380-386 (2010)

Sinha, B., A. Sarkar, P. Kumar y D. Brahman, Physicochemical Properties of L-Alanine in Aqueous silver Sulphate Solutions at $(298.15,308.15$ and 318.15$)$ K., doi:10.1007/s10765-011-1060-523, Int. J. Thermophys., 32(10), 20622078 (2011)

Wadi, R.K. y P. Ramasami, Partial molal Volumes and Adiabatic compressibilities of Transfer of Glycine and DL-Alanine from Water to Aqueous Sodium sulfate at 288.15, 298.15 and 308.15 K., doi:10.1039/A604650I, J. Chem. Soc. Faraday Trans., 93(2), 243-247 (1997)

Wang, J., Y. Zhenning, Z. Yang y C. Fengling, Partial molar Volumes and Viscosities of some Amino acids in Micellar Solutions of Sodium Caprylate, doi:10.1021/je049927v, J. Chem. Eng. Data., 49(5), 1354-1358 (2004)

Wang, X., F. Ranran, G. Yuhua y L. Ruisen, Volumetric Properties of Amino acids in Aqueous D-mannitol Solutions at 298.15 K., doi:10.1016/j.molliq.2014.04.028, J. Mol. Liq., 197, 73-76 (2014)

Xiaofeng, J., Z. Chunying y M. Youguang, Volumetric and Viscometric Studies of Amino acids in L -Ascorbic acid Aqueous Solutions at $\mathrm{T}=(293.15$ to 323.15$) \mathrm{K}$. , doi:10.1016/j.jct.2013.11.002, J. Chem. Thermodyn., 71, 50-63 (2014) 